\title{
Comparison of Functional Outcomes and Associated Complications in Patients Who Underwent Total Hip Arthroplasty for Femoral Neck Fracture in Relation to Their Underlying Medical Comorbidities
}

\author{
Byung Yoon Park, MD, Kuk Pil Lim, MD, Won Yong Shon, MD, PhD, \\ Y. Nishanth Shetty, MD, Ki Seong Heo, MD \\ Department of Orthopedic Surgery, Bumin Hospital, Busan, Korea
}

Purpose: In patients with independent mobility, full hip range of motion and sufficient muscle strength for daily life without cognitive impairment, treatment of a femoral neck fracture with total hip arthroplasty (THA) may be a better option compared to bipolar hip hemiarthroplasty. Here, functional outcomes and complications in patients who underwent THA for femoral neck fracture based on their comorbidity status were analyzed.

Materials and Methods: Between January 2013 and December 2018, 110 patients were treated with THA for femoral neck fractures at our institution. These patients were retrospectively analyzed for clinical outcomes at final follow-up (mean=24.4 months, range: 6-81 months) using the Harris hip score (HHS) and the presence or absence of two potential comorbidities: i) diabetes mellitus (DM; 35 with and 75 without) and ii) hypertension (HTN; 50 with and 60 without).

Results: The incidence of superficial infections at the surgical site in patients with DM was significantly higher compared with patients without DM $(P=0.024)$. There were no significant differences in other potential complications based on DM status. HHS at final follow-up between patients with and without DM and with and without HTN were not significantly different ( 83.3 vs. $81.0, P=0.39$ and 81.6 vs. $82.4, P=0.75$, respectively).

Conclusion: Superficial infections occurred more frequently in patients with DM compared with patients without DM. DM and HTN status are not correlated with HHS.

Key Words: Femoral neck fractures, Total hip arthroplasty, Comorbidity, Superficial infection

Submitted: September 16, 2019 1st revision: October 23, 2019

2nd revision: November 6, 2019 Final acceptance: November 12, 2019

Address reprint request to

Kuk Pil Lim, MD

[https://orcid.org/0000-0002-1247-4751]

Department of Orthopedic Surgery, Bumin Hospital, 59 Mandeok-

daero, Buk-gu, Busan 46555, Korea

TEL: +82-51-330-3082 FAX: +82-51-330-3075

E-mail: oskpilimahanmail.net
This is an Open Access article distributed under the terms of the Creative Commons Attribution Non-Commercial License (http://creativecommons. org/licenses/by-nc/4.0) which permits unrestricted non-commercial use, distribution, and reproduction in any medium, provided the original work is properly cited. 
Byung Yoon Park et al. Comparison between Patients with and without Medical Comorbidities Who Underwent THA

\section{INTRODUCTION}

The incidence of osteoporotic fractures is increasing due in part to an increase in the number of patients in the geriatric age group; the global incidence of hip fractures is expected to be about 6.26 million by $2050^{1-8}$. Total hip arthroplasty (THA) plays an important role in: i) reducing pain, ii) improving functional outcomes after surgery, iii) reducing the frequency of fixation failures, iv) enabling early weight bearing after surgery, and v) decreasing the frequency of complications (e.g., nonunion, leg shortening, femoral head osteonecrosis $)^{9-11}$. However, THA may result in a large volume of blood loss during surgery and increase operation times and the possibility of postoperative dislocations compared to hip osteosynthesis ${ }^{12}$. For these reasons, the general condition of a patients including comorbidities can significantly affect prognosis after $\mathrm{THA}^{13,14}$ including increased length of hospital stays and a higher probability of readmission in some cases ${ }^{15}$. It is well known that there is an increased incidence of superficial wound infections at the surgical site in patients with diabetes mellitus (DM) ${ }^{16-18)}$, however, few studies have assessed the potential correlation between functional outcomes and complications in Korean patients treated with THA for femur neck fractures. Here, patients who underwent THA, but not bipolar hip hemiarthroplasty were selected to exclude those with pre-surgery limitations of daily life and range of motion (ROM).

The purpose of this study was to compare functional outcomes and associated complications in patients with femoral neck fractures treated with THA potential comorbidity status (i.e., DM, hypertension [HTN]).

\section{MATERIALS AND METHODS}

\section{Study Design}

This retrospective study was approved by the Institutional Review Board (IRB) of Bumin Hospital (IRB No. 201909BM-007). One-hundred and ten patients who underwent THA for the treatment of femoral neck fractures between January 2013 and December 2018 were retrospectively analyzed. At the Bumin Hospital, THA, and not bipolar hip hemiarthroplasty, is performed on patients with independent mobility, no cognitive impairment, full hip ROM and sufficient muscle strength for daily life before femur neck fracture. Although age can be considered when choosing a surgical approach, we still prefer to perform THA (vs. bipolar hip hemiarthroplasty) for elderly patients who are healthy, have sufficient muscle strength and no dementia or gait disturbance. The approaches used were anterolaterally $(n=89)$ and posterolaterally $(n=21)$. Bencox ${ }^{\circledR}$ (Corentec, Seoul, Korea) acetabular cups and stems were used in 98 patients and VERILAST ${ }^{\mathrm{TM}}$ (Smith and Nephew, Memphis, TN, USA) in the remaining 13. Cementless fixation was used for all acetabular cups and femoral stems. For bearings, ceramic-on-ceramic systems $(n=84)$ and ceramic-onpolyethylene systems $(n=26)$ were used. Two surgeons performed surgeries with 62 conducted by one and 48 by the other. For head size, $36 \mathrm{~mm}(\mathrm{n}=109)$ and $38 \mathrm{~mm}$ $(n=1)$ were used. Soft hip abduction braces were applied immediately after surgeries. Postoperative rehabilitation consisted of quadriceps and hip abductor and flexor-muscle strengthening exercises; partial weight bearing with crutch ambulation was performed for six weeks postoperatively. We permitted light exercise (e.g., golf, bowling, jogging) after the sixth postoperative week.

Postoperative data was collected by electronic screening of the patients' electronic medical record and postoperative radiography. Inclusion criteria were: i) documented femoral neck fracture by referring orthopedic surgeon, ii) treatment with primary THA, and iii) normal gait and ROM of the injured hip joint before sustaining femoral neck fracture. Exclusion criteria were: i) primary THA for reasons other than femoral neck fracture, ii) treatment with revision THA, iii) dementia, and iv) gait disturbance and limitation of ROM of the injured hip joint before THA. Infections were identified with a validated surveillance electronic algorithm, and the results then were individually adjudicated by electronic chart review by a trained clinical research assistant. Baseline characteristics included demographics (age, sex), body mass index (BMI), and presence or absence of DM and HTN. Out of the 110 patients included, 35 were diabetic and 50 were hypertensive. No patient had both DM and HTN. During the same period, THAs were performed in 75 patients without DM. All patients were administered perioperative intravenous antibiotics typically until six days postoperatively. If a patient was diagnosed with a superficial infection, antibiotics were injected until CRP became normal and the infection was deemed under control. An indwelling urinary catheter was placed at the time of operation and was typically removed after two days unless otherwise indicated.

Functional outcomes (Harris hip score, HHS) and radiographic scores were evaluated preoperatively and postoperatively (eight weeks, six months, one year, and 
then two to three years thereafter). Complications were also recorded (e.g., superficial and deep infections, dislocation of the hip joint, aseptic loosening, periprosthetic fracture, deep vein thrombosis). We defined superficial and deep infection based on the Centers for Disease Control and Prevention or the Surgical Site Infection Surveillance Service criteria. Also, we distinguished between superficial infections and wound dehiscence ${ }^{19,20)}$.

\section{Statistical Analysis}

We used various statistical tools for analyses. For univariate analyses, the independent $t$-test was used for continuous variables (e.g., age, BMI). For categorical variables (e.g., sex) the chi-square test or Fisher's exact test was used. Multiple logistic regression analysis was performed to study the potential association between comorbidities and superficial infections after THA. To analyze the relationship between comorbidities and HHS, the independent $t$-test was applied. The IBM SPSS software package ver. 20.0 (IBM Corp., Armonk, NY, USA) was used for all statistical analyses, with the $\alpha$ level set at 0.05 .

\section{RESULTS}

One hundred and ten patients who underwent THA for femoral neck fractures were included. The mean age of patients was 68.7 years (range: $34-87$ years); 45 patients (40.9\%) and 65 patients (59.1\%) were male and female, respectively. A total of 35 patients had DM (17 males and 18 females); the remaining 75 patients did not have DM (28 males and 47 females). Mean BMI was $21.7 \mathrm{~kg} / \mathrm{m}^{2}$ (range: $16.4-28.8 \mathrm{~kg} / \mathrm{m}^{2}$ ) with an average follow-up period of 24.4 months (range: 6-81 months). The mean ages of patients with and without DM were 64.0 years (range: $34-$ 82 years) and 62.2 years (range: 61-87 years), respectively. Fifty patients had HTN (19 males and 31 females) and 60 patients did not have HTN ( 25 males and 35 females). The mean age of patients with and without HTN were 69.0 years (range: $70-87$ years) and 67.7 years (range: $34-82$ years), respectively. There were no significant differences in age, BMI or sex between groups broken out by DM or HTN status (Table 1).

Table 1. Demographic Data for Clinical Variables $(n=110)$

\begin{tabular}{|c|c|c|c|c|c|c|}
\hline \multirow{2}{*}{ Characteristic } & \multicolumn{6}{|c|}{ Potential comorbidity } \\
\hline & $D M(n=35)$ & Non-DM (n=75) & $P$-value & HTN $(n=50)$ & Non-HTN $(n=60)$ & $P$-value \\
\hline Age (yr) & $64.0 \pm 12.0$ & $62.2 \pm 9.8$ & 0.4 & $69.0 \pm 11.9$ & $67.7 \pm 10.3$ & 0.54 \\
\hline BMI $\left(\mathrm{kg} / \mathrm{m}^{2}\right)$ & $22.0 \pm 3.0$ & $21.5 \pm 2.9$ & 0.4 & $22.3 \pm 2.5$ & $21.3 \pm 3.2$ & 0.07 \\
\hline Sex & & & 0.26 & & & 0.7 \\
\hline Male & 17 & 28 & & 19 & 25 & \\
\hline Female & 18 & 47 & & 31 & 35 & \\
\hline
\end{tabular}

Values are presented as mean \pm standard deviation or number only. Statistical significances $(P<0.05)$ were tested by independent $t$-test for analysis of continuous variables (age and BMI), and the $\varkappa^{2}$ test or Fisher's exact test (sex) between groups.

DM: diabetes mellitus, HTN: hypertension, BMI: body mass index.

Table 2. Complications in the Patients with DM vs. without DM

\begin{tabular}{llccc}
\hline \hline Complication & $\mathrm{DM}^{+}$ & Non-DM & Multivariate OR (95\% CI) & $P$-value \\
\hline Superficial infection & $5 / 35$ & $1 / 75$ & $12.33(1.38-110.04)$ & $0.024^{*}$ \\
Aseptic loosening & $0 / 35$ & $0 / 75$ & - & - \\
Periprosthetic fracture & $0 / 35$ & $0 / 75$ & - & - \\
Neurologic deficit & $2 / 35$ & $0 / 75$ & $18.50(2.18-157.23)$ & $0.008 *$ \\
Total & $7 / 35$ & $1 / 75$ & & - \\
\hline
\end{tabular}

DM: diabetes mellitus, OR: odds ratio, $\mathrm{Cl}$ : confidence interval.

* Statistical significances $(P<0.05)$ were tested by multiple logistic regression analysis.

+ Subject number/total number. 
Byung Yoon Park et al. Comparison between Patients with and without Medical Comorbidities Who Underwent THA

\section{Comparison between Diabetic and Non-diabetic Patients}

The incidence of superficial surgical site infections was significantly higher in patients with DM (5/35 patients, $14.3 \%)$ compared with patients without DM (1/75 patients, $1.3 \%)(95 \%$ confidence interval $[\mathrm{CI}]=1.38-110.04, P=$ 0.024). Importantly, there were no significant difference in postoperative complications other than superficial infection. Dislocations, periprosthetic fracture, aseptic loosening and deep vein thrombosis were not observed in any patient. One patient (without DM) experienced polyethylene wear. Two patients with DM presented with neurologic deficit (sciatic nerve palsy $[n=1]$ and peroneal nerve palsy $[n=1])$; both resolved completely in 6 months (Table 2). There was no significant difference in HHS between the diabetic and non-diabetic groups at final follow-up (83.3 vs. 81.0, $P=0.39$ ) (Table 3).

\section{Comparison between Hypertension Group and Non-hypertension Group}

When comparing patients with and without $\mathrm{HTH}$, there was no significant difference in superficial infections (95\% $\mathrm{CI}=0.23-6.29, P=0.82$ ) (Table 4) or postoperative HHS (81.6 vs. $82.4, P=0.75$ ) (Table 3 ).

A single patient experienced polyethylene insert wear. No cases had hip dislocation, periprosthetic fracture, aseptic loosening, or deep vein thrombosis. Two patients without HTN experienced neurologic deficit.

\section{DISCUSSION}

Functional outcomes and complications in patients with femoral neck fracture treated with THA were analyzed in relation to comorbidity statuses. The aim of hip fracture surgery in the elderly is to remove pain and provide the best functional results while minimizing complications and revision rates ${ }^{21}$. Femoral neck fracture is a debilitating condition and associated comorbidities may increase institutional demands and hospital stay; research on this topic has been very limited in Korea. The most common complications of THA after femur neck fracture are dislocation $^{22}$, however no patients in our study experienced dislocation.

HHS was used to measure functional outcomes of the hip joint after THA. Due to the retrospective nature of the study, preoperative HHS could not be measured, hence no preoperative to postoperative comparison could be made. Although this is included as a limitation to this study, we note that only patients without gait disturbance or ROM limitations in the hip joint before surgery were included.

Previous studies have confirmed that comorbidities can affect prognoses after insertion of a prosthesis ${ }^{23-27)}$, especially in patients with DM who are at an increased risk for superficial and deep joint infection after arthroplasty ${ }^{16-18}$.

Table 3. Clinical Outcome in Patients with DM vs. without DM and HTN vs. without HTN

\begin{tabular}{lccc}
\hline \hline Harris hip score & Comorbidity & No-comorbidity & $P$-value \\
\hline DM & $83.3 \pm 12.5$ & $81.0 \pm 13.1$ & 0.39 \\
HTN & $81.6 \pm 13.3$ & $82.4 \pm 12.8$ & 0.75 \\
\hline
\end{tabular}

Values are presented as mean \pm standard deviation. Statistical significances $(P<0.05)$ were tested by independent $t$-test between groups.

DM: diabetes mellitus, HTN: hypertension.

Table 4. Complications in the Patients with HTN vs. without HTN

\begin{tabular}{lcccc}
\hline \hline Complication & HTN* & Non-HTN* & Multivariate OR (95\% CI) & $P$-value \\
\hline Superficial infection & $3 / 50$ & $3 / 60$ & $1.21(0.23-6.29)$ & 0.818 \\
Aseptic loosening & $0 / 50$ & $0 / 60$ & - & - \\
Periprosthetic fracture & $0 / 50$ & $0 / 60$ & - & - \\
Neurologic deficit & $0 / 50$ & $2 / 60$ & $0.70(0.16-3.10)$ & 0.640 \\
Total & $3 / 50$ & $5 / 60$ & - \\
\hline
\end{tabular}

Statistical significances $(P<0.05)$ were tested by multiple logistic regression analysis.

HTN: hypertension, OR: odds ratio, $\mathrm{Cl}$ : confidence interval.

* Subject number/total number. 
Maradit Kremers et al. ${ }^{28)}$ and Song et al. ${ }^{14)}$ demonstrated that: i) DM was a significant risk factor for prosthetic joint infections and ii) DM was a risk factor for surgical site infection in a cohort study of 20,171 total hip and knee arthroplasty procedures ${ }^{28}$ and a retrospective cohort study, respectively ${ }^{14}$. In a study by Jain et al. ${ }^{29}$, an increase in postoperative complications in patients with DM who underwent THA and total knee arthroplasty (TKA) was observed. These results were similar to the results in this study and those of several previous studies involving patients with $\mathrm{DM}^{30-33}$. Here, we observed an increase in the incidence of superficial infection in patients with DM compared to those without DM. Hence, extra precautions must be taken in patients with DM who are undergoing THA.

Although Chun et al ${ }^{34)}$ reported an increased incidence of deep infection in patients with DM in their study of 616 patients who underwent THA in Korea, similar results were not achieved here ${ }^{35}$. One potential reason for this difference may be the relatively small sample size of this analysis, a noted limitation of this study. There were no cases of deep infection in this study, an observation that suggests that more cases may be required to analyze this potential complication; further studies can be designed to address this limitation.

Based on the connection between DM status and superficial infections at the surgical site and the relatively high frequency of patients with inadequately controlled blood sugars, we suggest that a targeted precautionary approach to check blood glucose during the perioperative period should be mandatory. However, due to the urgent need for surgery in patients with femoral neck fractures in order to avoid poor prognosis and failure of surgery, it may be difficult to take time to control DM and HTN in the perioperative period.

In this study there was also no association of postoperative complications in the presence of systemic HTN, a result similar to that published by Perka et al. ${ }^{35}$ and Freedman et al. ${ }^{36)}$ who evaluated patients who underwent TKA and THA, respectively ${ }^{36)}$.

In some previous studies, DM has been recognized as a factor that increases the incidence of superficial infections in the wound and inhibits healing ${ }^{16-18}$; superficial infections after THA were also related to DM status in this study. However, DM was not significantly associated with complications other except superficial infections and HTN status was also not significantly associated with complications and functional prognosis of hip joints. HTN is one of the most common and important comorbidities and should be considered when THA for femoral neck fractures are performed, however, HTN had no significant impact on complications and functional outcome assessed here. Importantly, these findings do not mean that DM and HTN should not be strictly controlled preoperatively and postoperatively. Because femoral neck fracture usually require an urgent surgery, delayed operations may lead to failure of the operation and/or poor prognosis. Thus, both criteria (i.e., control of comorbidity, timely surgery) should always be simultaneously considered.

This study demonstrated that DM was a risk factor for superficial infections, however, there were no cases of deep infections here and thus the relationship between comorbidities and outcomes (i.e., all potential complications, HHS) could not be fully validated. Additional studies will help characterize the potential for comorbidities to serve as risk factors for deep infection of the hip joint after THA.

The short follow-up period (i.e., one year) is another limitation of this study. A significant number of patients are often lost to follow-up due to their old age preventing us from securing follow-up beyond one year. Therefore, the results presented here may not properly reflect longterm outcomes; additionaly studies are being planned to assess this possibility in the future.

\section{CONCLUSION}

In patients with DM, superficial infections occurred more frequently compared with patients without DM; HTN was not correlated with superficial infections. There was also no correlation between comorbidities and complications other than superficial infections.

\section{CONFLICT OF INTEREST}

The authors declare that there is no potential conflict of interest relevant to this article.

\section{REFERENCES}

1. Bozic KJ, Ries MD. The impact of infection after total hip arthroplasty on hospital and surgeon resource utilization. J Bone Joint Surg Am. 2005;87:1746-51.

2. Ridgeway S, Wilson J, Charlet A, Kafatos G, Pearson A, Coello R. Infection of the surgical site after arthroplasty of the hip. J Bone Joint Surg Br. 2005;87:844-50.

3.Bozic KJ, Ward DT, Lau EC, et al. Risk factors for periprosthetic joint infection following primary total hip arthroplasty: a case control study. J Arthroplasty. 2014; 29:154-6.

4. Poultsides LA, Ma Y, Della Valle AG, Chiu YL, Sculco TP, 
Byung Yoon Park et al. Comparison between Patients with and without Medical Comorbidities Who Underwent THA

Memtsoudis SG. In-hospital surgical site infections after primary hip and knee arthroplasty: incidence and risk factors. J Arthroplasty. 2013;28:385-9.

5. Dale H, Fenstad AM, Hallan G, et al. Increasing risk of prosthetic joint infection after total hip arthroplasty. Acta Orthop. 2012;83:449-58.

6. Everhart JS, Altneu E, Calhoun JH. Medical comorbidities are independent preoperative risk factors for surgical infection after total joint arthroplasty. Clin Orthop Relat Res. 2013; 471:3112-9.

7. Namba RS, Inacio MC, Paxton EW. Risk factors associated with surgical site infection in 30,491 primary total hip replacements. J Bone Joint Surg Br. 2012;94:1330-8.

8. Kannus P, Parkkari J, Sievänen H, Heinonen A, Vuori I, Järvinen M. Epidemiology of hip fractures. Bone. 1996;18: $57 S-63 S$.

9. Avery PP, Baker RP, Walton MJ, et al. Total hip replacement and hemiarthroplasty in mobile, independent patients with a displaced intracapsular fracture of the femoral neck: a seven- to ten-year follow-up report of a prospective randomised controlled trial. J Bone Joint Surg Br. 2011;93:1045-8.

10. Lai K, Bohm ER, Burnell C, Hedden DR. Presence of medical comorbidities in patients with infected primary hip or knee arthroplasties. J Arthroplasty. 2007;22:651-6.

11. Pulido L, Ghanem E, Joshi A, Purtill JJ, Parvizi J. Periprosthetic joint infection: the incidence, timing, and predisposing factors. Clin Orthop Relat Res. 2008;466:1710-5.

12. van Vugt AB, Oosterwijk WM, Goris RJ. Osteosynthesis versus endoprosthesis in the treatment of unstable intracapsular hip fractures in the elderly. A randomised clinical trial. Arch Orthop Trauma Surg. 1993;113:39-45.

13. Bozic KJ, Lau E, Kurtz S, et al. Patient-related risk factors for periprosthetic joint infection and postoperative mortality following total hip arthroplasty in Medicare patients. J Bone Joint Surg Am. 2012;94:794-800.

14. Song KH, Kim ES, Kim YK, et al. Differences in the risk factors for surgical site infection between total hip arthroplasty and total knee arthroplasty in the Korean Nosocomial Infections Surveillance System (KONIS). Infect Control Hosp Epidemiol. 2012;33:1086-93.

15. Paxton EW, Inacio MC, Singh JA, Love R, Bini SA, Namba RS. Are there modifiable risk factors for hospital readmission after total hip arthroplasty in a US healthcare system? Clin Orthop Relat Res. 2015;473:3446-55.

16. Parvizi J, Gehrke T, Chen AF. Proceedings of the international consensus meeting on periprosthetic joint infection. Foreword. J Orthop Res. 2014;32 Suppl 1:S2-3.

17. Golden SH, Peart-Vigilance C, Kao WH, Brancati FL. Perioperative glycemic control and the risk of infectious complications in a cohort of adults with diabetes. Diabetes Care. 1999;22:1408-14.

18. Moghissi ES, Korytkowski MT, DiNardo M, et al. American Association of Clinical Endocrinologists and American Diabetes Association consensus statement on inpatient glycemic control. Endocr Pract. 2009; 15:353-69.

19. Horan TC, Gaynes RP, Martone WJ, Jarvis WR, Emori TG. CDC definitions of nosocomial surgical site infections, 1992: a modification of CDC definitions of surgical wound infections. Infect Control Hosp Epidemiol. 1992;13:606-8.
20. Ousey K, Djohan R, Dowsett C, et al. Surgical wound dehiscence: improving prevention and outcomes. Wounds UK 2018; 1:48.

21. Travis EC, Tan RS, Funaki P, McChesney SJ, Patel SC, Brogan K. Clinical outcomes of total hip arthroplasty for fractured neck of femur in patients over 75 years. J Arthroplasty. 2015;30:230-4.

22. Berry DJ, von Knoch M, Schleck CD, Harmsen WS. Effect of femoral head diameter and operative approach on risk of dislocation after primary total hip arthroplasty. J Bone Joint Surg Am. 2005;87:2456-63.

23. van Kasteren ME, Manniën J, Ott A, Kullberg BJ, de Boer AS, Gyssens IC. Antibiotic prophylaxis and the risk of surgical site infections following total hip arthroplasty: timely administration is the most important factor. Clin Infect Dis. 2007;44:921-7.

24. Joshi N, Caputo GM, Weitekamp MR, Karchmer AW. Infections in patients with diabetes mellitus. $N$ Engl J Med. 1999; 16:1906-12.

25. Eibl N, Spatz M, Fischer GF, et al. Impaired primary immune response in type-1 diabetes: results from a controlled vaccination study. Clin Immunol. 2002;103(3 Pt 1):249-59.

26. Pozzilli $\mathrm{P}$, Gale EA, Visalli N, et al. The immune response to influenza vaccination in diabetic patients. Diabetologia. 1986;29:850-4.

27. Marchant MH Jr, Viens NA, Cook C, Vail TP, Bolognesi MP. The impact of glycemic control and diabetes mellitus on perioperative outcomes after total joint arthroplasty. $J$ Bone Joint Surg Am. 2009;91:1621-9.

28. Maradit Kremers H, Lewallen LW, Mabry TM, Berry DJ, Berbari EF, Osmon DR. Diabetes mellitus, hyperglycemia, hemoglobin AlC and the risk of prosthetic joint infections in total hip and knee arthroplasty. J Arthroplasty. 2015; 30:439-43.

29. Jain NB, Guller U, Pietrobon R, Bond TK, Higgins LD. Comorbidities increase complication rates in patients having arthroplasty. Clin Orthop Relat Res. 2005;(435):232-8.

30. England SP, Stern SH, Insall JN, Windsor RE. Total knee arthroplasty in diabetes mellitus. Clin Orthop Relat Res. 1990;(260):130-4.

31. Forrest G, Fuchs M, Gutierrez A, Girardy J. Factors affecting length of stay and need for rehabilitation after hip and knee arthroplasty. J Arthroplasty. 1998;13:186-90.

32. Serna F, Mont MA, Krackow KA, Hungerford DS. Total knee arthroplasty in diabetic patients. Comparison to a matched control group. J Arthroplasty. 1994;9:375-9.

33. Yang K, Yeo SJ, Lee BP, Lo NN. Total knee arthroplasty in diabetic patients: a study of 109 consecutive cases. $J$ Arthroplasty. 2001;16:102-6.

34. Chun YS, Lee SH, Lee SH, Cho YJ, Rhyu KH. Clinical implication of diabetes mellitus in primary total hip arthroplasty. Hip Pelvis. 2014;26:136-42.

35. Perka C, Arnold U, Buttgereit F. Influencing factors on perioperative morbidity in knee arthroplasty. Clin Orthop Relat Res. 2000;(378):183-91.

36. Freedman DS, Khan LK, Serdula MK, Galuska DA, Dietz WH. Trends and correlates of class 3 obesity in the United States from 1990 through 2000. JAMA. 2002;288:1758-61. 